\title{
Proximal optimization technique, proximal rewiring, side-branch dilation and paired balloon inflation to minimize metal carina in inadequate jailing
}

\author{
Fumiaki Nakao \\ Department of Cardiology, Yamaguchi Grand Medical Center, Yamaguchi, Japan
}

If distal rewiring is selected for inadequate jailing (distal cell on a side-branch ostium divided by a stent-link) during single two-link stent deployment at the coronary bifurcation with side-branch dilation, kissing balloon inflation (KBI) might result in residual deformed struts in the side-branch ostium, and a sparse strut-covered distal main vessel. Although adequate rewiring during inadequate jailing might be proximal rewiring [Nakao $\mathrm{F}$.
The impact of proximal cell rewiring compared with distal cell rewiring with single-stent deployment and subsequent kissing balloon inflation in the inadequate jailing of a Kanam stent on the side branch ostium. Cardiol J. 2017 Jun 27; ahead of print], simple KBI might result in residual metal carina. The sequence of procedures should also be considered.

A SYNERGY ${ }^{\mathrm{TM}}$ two-link stent (Boston Scientific Co.) was deployed in the main vessel of a bifurca-

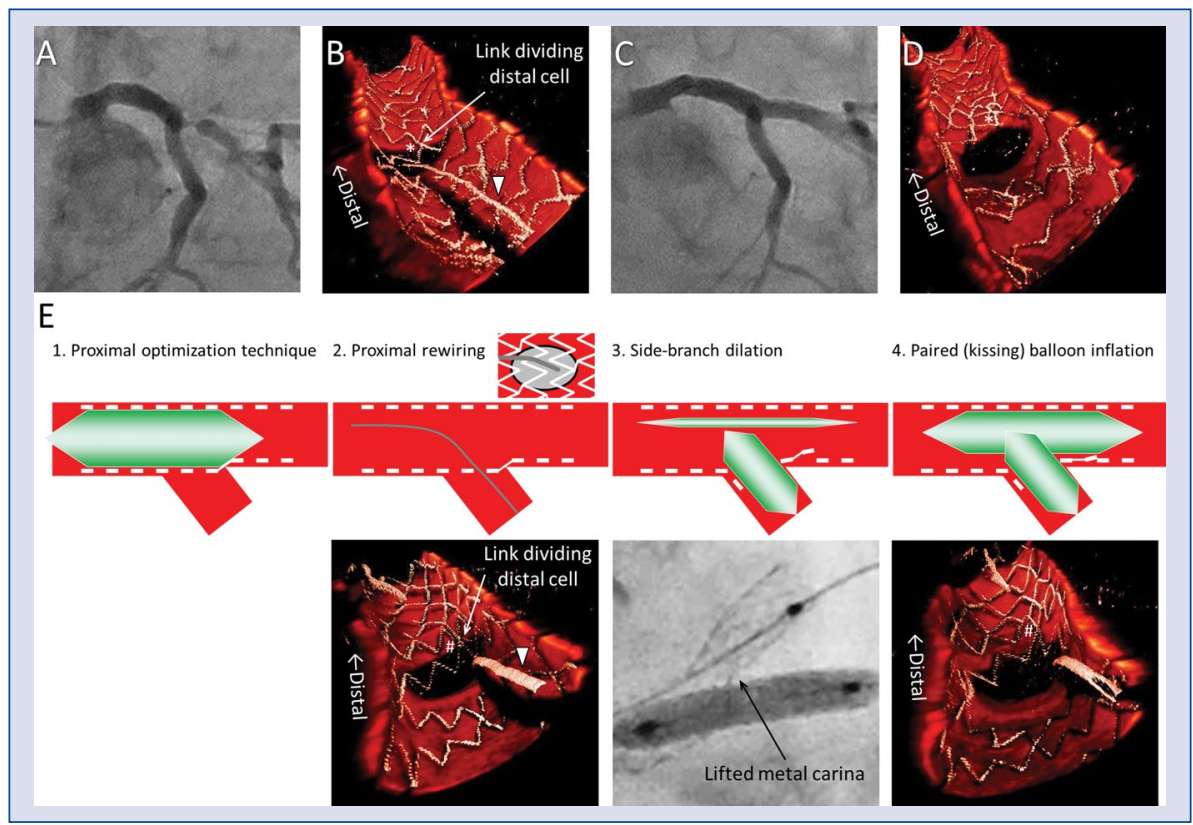

Figure 1. Clinical case with SYNERGY ${ }^{\mathrm{TM}}$ and schema of PPSP procedure. A. Baseline coronary angiogram; B. Longitudinal 3D-OCT cut-away view of inadequate jailing and proximal rewiring; C. Final coronary angiogram; D. Final 3D-OCT image; E. Schema of PPSP procedure with X-ray fluorogram and 3D-OCT image of case with two-link BMX-J ${ }^{\mathrm{TM}}$ stent (Biosensors International Group Ltd.); ${ }^{*}$ and ${ }^{*}$, same links divide distal cell at rewiring; arrowhead, re-crossed wire.

Address for correspondence: Dr. Fumiaki Nakao, Department of Cardiology, Yamaguchi Grand Medical Center, 77 Ohsaki, Hofu, Yamaguchi 747-8511, Japan, tel: +81-835-22-4411, fax: +81-835-38-2210, e-mail: nakao-ymghp@umin.ac.jp 
tion lesion (Fig. 1A) and the proximal optimization technique (POT) was applied. Three-dimensional optical coherence tomography (3D-OCT) confirmed inadequate jailing and proximal rewiring (Fig. 1B). Side-branch dilation was followed by KBI (paired balloon inflation, Fig. 1C). The final 3D-OCT showed a shift to the distal main vessel and minimization of the metal carina (Fig. 1D).

Figure $1 \mathrm{E}$ shows a schema of the POT, proximal rewiring, side-branch dilation, and paired balloon inflation (PPSP) procedure with X-ray fluorogram and 3D-OCT images of a patient with a different two-link stent. Such stents are weak longitudinally, and their struts can be moved longitudinally if properly handled. POT tilts the metal carina to the side-branch side, proximal rewiring and side-branch dilation lift the metal carina to the distal main vessel, and KBI apposes struts to the vessel wall. The PPSP procedure might help to minimize the metal carina when jailing by a twolink stent is inadequate. Further clinical studies are required.

Conflict of interest: None declared 